\title{
Tabletop imaging of structural evolutions in chemical reactions
}

\author{
Heide Ibrahim ${ }^{1}$, Benji Wales ${ }^{2}$, Samuel Beaulieu ${ }^{1}$, Bruno E. Schmidt ${ }^{1}$, Nicolas Thiré ${ }^{1}$, Éric \\ Bisson $^{1}$, Christoph T. Hebeisen ${ }^{3,4}$, Vincent Wanie ${ }^{1}$, Mathieu Giguére ${ }^{1}$, Jean-Claude Kieffer ${ }^{1}$, \\ Joseph Sanderson ${ }^{2}$, Michael S. Schuurman ${ }^{3}$, François Légaré $^{1}$ \\ 1. Institut National de la Recherche Scientifique, Centre Énergie Matériaux et Télécommunications, 1650 Boulevard Lionel- \\ Boulet, Varennes, Qc, J3X1S2, Canada \\ 2. Department of Physics and Astronomy, University of Waterloo, 200 University Avenue West, Waterloo, ON N2L 3G1, \\ Canada \\ 3. National Research Council of Canada, 100 Sussex Dr., Ottawa, K1A OR6, Canada \\ 4. Department of Physics, University of Ottawa, 150 Louis Pasteur, Ottawa ON, K1N 6N5, Canada
}

nuclear and electronic evolution of a molecule in time and space as it undergoes a chemical reaction. Using Coulomb Explosion Imaging we have shot the first highresolution molecular movie of a to and fro isomerization process in the acetylene cation. So far, this kind of phenomenon could only be observed using VUV light from a Free Electron Laser [Phys. Rev. Lett. 105, 263002 (2010)]. Here we show that 266 nm ultrashort laser pulses are capable of initiating rich dynamics through multiphoton ionization. With our generally applicable tabletop approach that can be used for other small organic molecules, we have investigated two basic chemical reactions simultaneously: proton migration and $\mathrm{C}=\mathrm{C}$ bond-breaking, triggered by multiphoton ionization. The experimental results are in excellent agreement with the timescales and relaxation pathways predicted by new and definitively quantitative ab initio trajectory simulations. 
Since the introduction of femto-chemistry ${ }^{1-5}$, electron or X-ray diffraction have been the

27 most commonly employed techniques to track nuclear rearrangement in molecules ${ }^{6-9}$.

28 Unfortunately, these techniques are largely insensitive to the more subtle and irregular structural

29 changes that can occur within a single small molecule undergoing a chemical reaction. Pump-

30 probe Coulomb Explosion Imaging (CEI) allows observation of these changes on a femtosecond

31 (fs) timescale with atomic resolution ${ }^{10-13}$. So far, however, important phenomena such as proton

32 migration in the acetylene cation, have only been observed using VUV light from a Free

33 Electron Laser $(\mathrm{FEL})^{14}$. It will be presented here that results obtained in a tabletop multiphoton approach can go well beyond those FEL results, including a high resolution molecular movie of proton migration from the linear acetylene cation $\left([\mathrm{HC}=\mathrm{CH}]^{+}\right)$to the vinylidene cation $\left(\left[\mathrm{C}=\mathrm{CH}_{2}\right]^{+}\right)$.

Since laser driven tunnel ionization preferentially ionizes a neutral molecule to the

37 electronic ground state of the cation, any subsequent nuclear dynamics will generally occur on that state. The ability to readily initiate dynamics on an excited electronic state of the cation would enable a variety of new and complex experiments. Employing the intuitive Koopman's approximation picture of ionization ${ }^{15}$, the ground electronic state of a cation is generated by

41 removal of an electron from the highest molecular orbital (HOMO) of the neutral, while excited

42 states are generated by ionizing electrons from the lower-lying HOMO-n orbitals. It had for 43 example been assumed that only ionization of the HOMO contributes to High Harmonic

44 Generation (HHG) - because of the low frequency fields used. However, recent results, 45 obtained at $800 \mathrm{~nm}$, demonstrate that ionization of HOMO-n orbitals may also contribute to an $46 \mathrm{HHG}$ spectrum ${ }^{16-18}$. Given the coherent nature of HHG process, even a small excited state 47 contribution can have a significant impact on the observed signal due to interference effects.

48 However, in CEI these contributions add linearly, and thus a small contribution from ionization of 49 the HOMO-n would be difficult to observe. 
To efficiently launch dynamics in excited states of molecules with a large energy gap

51 between ground and excited states it is common to use high photon energy sources such as

52 FELs (acetylene) ${ }^{14}$ or HHG (ethylene) ${ }^{19}$. If sub-fs resolution is required, one can use an XUV

53 pump/XUV probe arrangement based on HHG for this kind of experiments ${ }^{20,21}$, however at the

54 price of a highly demanding experimental setup. Here we show that multiphoton ionization with

55 ultrashort UV laser pulses provides a powerful alternative to demanding VUV sources.

Even though acetylene $\left(\mathrm{C}_{2} \mathrm{H}_{2}\right)$ has been serving as a model system for decades, proton

57 migration dynamics in its cation is not yet fully elucidated and has recently attracted attention of both experimental and theory groups ${ }^{12-14,22-24}$. Given its small size, it is an ideal candidate for the application of quantitative electronic structure theory, which can be brought to bear on its ground and excited states. dication of deuterated acetylene by Hishikawa and coworkers ${ }^{12,13}$. Very recently, optimal control experiments confirmed that excitation with $800 \mathrm{~nm}$ pulses leads to isomerization in the dication ${ }^{25}$.

64 These $800 \mathrm{~nm}$ pump schemes, however, are not capable of launching proton migration in other 65 charge states such as in the cation ${ }^{26}$.

67 which separates the acetylene cation in its ground state $X^{2} \Pi_{u}$ from vinylidene, as indicated in 68 the experimental scheme of Figure 1. Therefore, to obtain sufficient energy to overcome this 69 isomerization barrier ${ }^{22}$, it is necessary to populate the $\mathrm{A}$-state $\left(\mathrm{A}^{2} \Sigma_{\mathrm{g}}{ }^{+}\right)$of $[\mathrm{HC}=\mathrm{CH}]^{+}(5.8 \mathrm{eV}$ above the ground state), which requires ionization from the HOMO-1. This cannot be achieved

71 in the adiabatic regime with $800 \mathrm{~nm}$ pulses $^{26}$. To remain in the adiabatic regime for long

72 conjugated molecules that display small energy gaps, Lezius et al. ${ }^{28}$ have suggested increasing 73 the laser wavelength. Inverting this idea, it appears sensible to reach the non-adiabatic regime 
74 for small molecules by employing UV wavelengths with larger photon energies. Here we present a generalized approach using $266 \mathrm{~nm}$ pump pulses that unites the convenience and flexibility of a tabletop setup with excitation ranges of FELs - and unveils the isomerization process in unprecedented detail allowing us to record a molecular movie. This movie shows for the first time, that multiple oscillations can occur between the two isomers acetylene and vinylidene.

This paper is structured as follows: Firstly, in a single pulse experiment we verify that excitation with $266 \mathrm{~nm}$ pulses leads to the same spectral signatures as those obtained with an $\mathrm{FEL}^{14}$, confirming that we populate the first excited electronic state of the acetylene cation $\left(\mathrm{A}^{2} \Sigma_{\mathrm{g}}{ }^{+}\right)$which initiates proton migration. Secondly, we present time-resolved pump probe experiments of the two-body breakup. Based on the single-pulse experiments, the presence of $\mathrm{CH}_{2}{ }^{+}$in the dynamics of the vinylidene channel in the cation $\left(\mathrm{C}^{+}+\mathrm{CH}_{2}{ }^{+}\right.$correlation $)$is a clear indicator of proton migration since one proton has been migrating to the other side of the molecule. In addition to proton migration resulting in the formation of bound vinylidene molecules in the cation, new channels that lead to dissociation of the cation are observed in both, the vinylidene and the acetylene $\left(\mathrm{CH}^{+}+\mathrm{CH}^{+}\right)$correlations. And thirdly, we investigate the three-fragment correlations from the same experimental data set $\left(\mathrm{C}^{+}+\mathrm{CH}^{+}+\mathrm{H}^{+}\right)$and construct a molecular movie of proton migration, which exhibits excellent agreement with new numerical simulations of nonadiabatic wavepacket propagation on the ground and excited electronic states of the cation. These ab initio trajectory simulations employ definitive levels of electronic structure theory in the description of the relevant potential energy surfaces.

SINGLE PULSE EXPERIMENT. Signatures of proton migration in $\mathrm{C}_{2} \mathrm{H}_{2}{ }^{+}$have recently been demonstrated at the FEL in Hamburg ${ }^{14}$ with XUV light (38 eV). Indications of time dependent acetylene - vinylidene isomerization, photoinitiated via ionization to the A-state of the acetylene cation appear in the kinetic energy release (KER) of correlated $\mathrm{C}^{+}$and $\mathrm{CH}_{2}^{+}$ fragments at energies above $5.8 \mathrm{eV}^{14}$. As an initial experiment we set out to find this signature 
by employing single laser pulses of $266 \mathrm{~nm}$ central wavelength and varying pulse duration and peak power. The experimental scheme in terms of relevant electronic and ionic states and excitation pathways is described in Figure 1. The A-state of the acetylene cation is reached by absorption of four $266 \mathrm{~nm}$ photons (corresponding to $4 \times 4.66 \mathrm{eV}=18.64 \mathrm{eV}$ ). Once on the A-state, proton migration occurs, which is then followed by ionization to a dissociative state of the dication within the same laser pulse. Results of a single $266 \mathrm{~nm}$ pulse experiment are shown in Figure 2a), containing the KER distributions of the two-fragment correlation channel of vinylidene for 32 fs (green and red), 110 fs (black) and $250 \mathrm{fs}$ (blue) pulse duration and various pulse powers. Already a short pulse of moderate pulse power (green solid line) leads to a KER distribution broadened on the high energy side. With increasing pulse duration a strongly pronounced high-energy shoulder appears. The shoulder obtained with 250 fs pulses (blue solid line) agrees very well with data obtained at the FEL using $38 \mathrm{eV}$ (open circles) ${ }^{14}$. This shoulder was assigned to proton migration initiated by ionizing acetylene to the A-state of its cation $^{14,22,24,29}$. The proton migration which we observe is essentially pulse duration dependent and only secondarily intensity dependent. The pulse powers of the short 32 fs pulse $\left(1 \cdot 10^{8} \mathrm{~W}\right.$ and $2 \cdot 10^{8} \mathrm{~W}$ ), which are higher by a factor of five than those for the long pulse, indicate that increasing the intensity alone will not lead to a strongly pronounced high energy shoulder. Fragmentation occurs in the doubly charged state which is accessed here in a sequential process within a single pulse. This effect is therefore most pronounced for the longest pulses (250 fs) which are also of the lowest power.

Additionally, we employed $800 \mathrm{~nm}, 200$ fs pulses (grey shaded). The energetically narrow KER distribution from $800 \mathrm{~nm}$ excitation confirms the observation of Alnaser et al. ${ }^{26}$. In this case, we do not obtain high energy fragments as a signature of isomerization in the acetylene cation and similar results were obtained at $400 \mathrm{~nm}$ (not shown). This was a primary 
motivation for our study at $266 \mathrm{~nm}$ in order to confirm that UV excitation is essential to populate the A-state.

INITIAL STATE ASSIGNMENT - I. Assignment of the electronic states involved in the isomerization can be more demanding in a multiphoton scheme compared to a single photon scheme pumped by one VUV photon. In both cases however, multiple states can be populated, e.g. the ground and the first excited state of the cation. In our experiment, while the cationic Astate is excited by four photons, the ground state of the cation $X^{2} \Pi_{u}$ is populated in a three photon process. However, the documented energy barrier separating the acetylene and vinylidene isomers will prohibit proton migration from low-lying vibrational levels on the electronic ground state ${ }^{27}$, so its contribution will only show up around the main peak of KER in Figure 2a). To confirm that the experimentally observed high energy shoulder arises from ionization to the A-state ${ }^{14}$, we investigate the angular distribution of the molecular fragments from the KER spectrum obtained with the $110 \mathrm{fs,} 266 \mathrm{~nm}$ pulse shown as a black solid line in Figure 2a). Initially, all population is in the $\mathrm{X}^{1} \Sigma_{g}^{+}$electronic state of the neutral acetylene. Following Koopman's approximation ${ }^{15}$, ionization to the $\mathrm{A}^{2} \Sigma_{g}{ }^{+}$state of the acetylene cation requires the removal of one electron from the $\mathrm{HOMO}-1$, which is a $\sigma_{\mathrm{g}}$ orbital. The probability for ionization from this orbital is largest for those molecules aligned parallel to the polarisation of the laser field ${ }^{17}$ ( this corresponds to the angle $\theta=0^{\circ}$, consistent with the definition given in the inset of Figure 1). Note that this is exactly where the maximum in the high energy shoulder of the angular resolved KER distribution is observed (see cyan shaded curve of Figure $2 b$ ). The high energy maximum of this particular angular slice is significantly more pronounced than for all other angular distributions and even exceeds the main peak. Thus, we can confidently assign this region to ionization to the $\mathrm{A}^{2} \Sigma_{g}{ }^{+}$state. Although higher electronic states with $\Sigma$ symmetry exist in the cation, the probability of them being populated under the current experimental 
condition is significantly lower than for the A-state (see section II. in the supplementary information $-\mathrm{SI})$.

PUMP PROBE EXPERIMENT - TWO BODY BREAKUP. Next, we employed pump-probe CEI to image the dynamics resulting from multiphoton ionization with $266 \mathrm{~nm}$ pulses. We investigate two chemical reactions: i) proton migration - one of the fundamental processes in chemistry and biology, and ii) the first observation of $\mathrm{C}=\mathrm{C}$ bond breaking on highly excited states of the acetylene and vinylidene cation.

We focus first on the isomerization process: As shown in the experimental scheme of Figure 1, a $266 \mathrm{~nm}$ pump-pulse ionizes the system to $[\mathrm{HC}=\mathrm{CH}]^{+}$in a four-photon process thereby initiating proton migration dynamics. A time delayed, $800 \mathrm{~nm}$ probe-pulse tunnel-ionizes the molecule to doubly and triply charged states of acetylene or vinylidene. Its electric field stripes off electrons, leaving behind positively charged fragments which undergo Coulomb explosion. As in the single pulse experiments, proton migration dynamics shows up in pumpprobe experiments in the high-energy region obtained by correlating two fragments. This dynamics corresponds to the region V3 of the vinylidene channel $\left(\mathrm{C}^{+}+\mathrm{CH}_{2}^{+}\right)$in Figure 3a). We observe an increase of the yield in this high energy shoulder in the temporal window of 60-100 fs followed by a decrease. (We will leave the details about the proton migration dynamics and vinylidene formation until we have direct evidence from three fragment correlations of Figure 5 and Figure 6.) Note that this high energy shoulder is less pronounced in the pump-probe experiment of Figure 3 compared to the single pulse experiment in Figure 2a), in which the complete two-step ionization process occurs many times beneath a single pulse envelope. Therefore, for long single pulse durations (110 and $250 \mathrm{fs}$ ) the excited state dynamics can be initiated on a larger number of molecules and this gives rise to an accumulated vinylidene formation, compared to the $32 \mathrm{fs}$ in case of the pump-probe experiment. 
The mid-energy region V2 in Figure $3 a$ ) has been previously ${ }^{14}$ assigned to a

172 sequential double ionization. In addition we observe a new peak emerging in the low-energy

173 region $\mathrm{V} 1$. This peak is first visible around $80 \mathrm{fs}$, and has, to our knowledge, never been

174 observed before for $\mathrm{C}_{2} \mathrm{H}_{2}{ }^{+}$. Since it occurs for both vinylidene and acetylene $\left(\mathrm{CH}^{+}+\mathrm{CH}^{+}\right.$, Figure

$1753 b)$, we attribute it to a stretching of the $C=C$ bond. In both channels it migrates to smaller

176 energies (indicating an increase in the C-C internuclear distance) with increasing time delay.

177 The peak strength continuously increases with increasing time delay, which is consistent with a 178 lowering of the ionization potential as the $\mathrm{C}=\mathrm{C}$ bond length increases. elongation of the $\mathrm{C}=\mathrm{C}$ bond to $>4 \mathrm{r}_{\mathrm{eq}}$, where $\mathrm{r}_{\mathrm{eq}}$ is the equilibrium distance, implying that the

181 molecule has largely dissociated. Similar dissociation effects have been observed in $\mathrm{C}_{2} \mathrm{H}_{2}{ }^{2+} 30$

182 and 1,3-butadiene ${ }^{31}$. While none of the low lying electronic states of $\mathrm{C}_{2} \mathrm{H}_{2}{ }^{+}$is dissociative along 183 the C-C stretch coordinate, if a fifth photon is absorbed during the pump-pulse, the molecule will 184 have energy in excess of the lowest C-C dissociation asymptotes (see Figure 1 and Figure S9 185 in the SI). Since the dissociation dynamics occur in both the acetylene and vinylidene channels 186 they are likely to be triggered by the same event, namely absorption of a photon to higher lying 187 excited states of the cation. From there it can proceed to dissociative states of the vinylidene 188 cation. This dissociation pathway has not been observed at a FEL ${ }^{14}$ given that for one-photon 189 absorption, once ionized, no further coupling between electronic states occurs. We predict that 190 in a multiphoton experiment with shorter pump-pulses this sequential coupling after ionization is 191 also likely to be suppressed.

THREE BODY BREAKUP. This new dissociation channel also shows up in the three-body 193 breakup $\mathrm{C}_{2} \mathrm{H}_{2}{ }^{3+} \rightarrow \mathrm{H}^{+}+\mathrm{C}^{+}+\mathrm{CH}^{+}$, in which two electrons are removed by the probe pulse. Before 194 we address the rich information present in this channel though, we first need to prove that it is 195 indeed what we claim and that the probe pulse is only showing us dynamics of the cation. As a 
first step in doing so, we compare the KER features in the two-body channel (Figure 3), which

197 we have established to show us time dependent processes which must originate in the cation,

198 with those of the three-body channel in Figure 4. Panel a) contains the total kinetic energy

199 released during the Coulomb explosion. The peak maximum migrates from approximately $15 \mathrm{eV}$

200 at time zero to $7 \mathrm{eV}$ at a time delay of a picosecond, indicating the presence of the dissociative

201 channel described in the previous section. To uncover the close analogy between two- and

202 three-body breakup we take advantage of the fact that in the three-body channel an $\mathrm{H}^{+}$fragment

203 is created, which, because of conservation of momentum, is responsible for carrying away most

204 of the increased and broadband energy release. If the 3+ channel is observing the same

205 dynamics, we expect strong similarities in the energy sum of correlated $\mathrm{C}^{+}+\mathrm{CH}^{+}$fragments of

206 the three-body channel, with the total energy released in the two-body channel. To extract

207 hidden sub-structures of Figure 4a) we look at the 3+ KER reduced by the proton energy in b),

208 leading to a double peak structure with one peak around $6 \mathrm{eV}$ and another one emerging for longer times and moving to $1 \mathrm{eV}$ at one picosecond. The overall KER is still slightly higher compared to the two-body breakup since here Coulomb potentials are more closely

211 approximated. The key difference being that while both of the main peaks are well separated in

212 the two-body case, the peaks in Figure 4b) are much broader, leading to an overlap of the 213 dominant spectral features. This is due to the additional broadband energy both, the $\mathrm{CH}^{+}$, and 214 the $\mathrm{C}^{+}$fragments gather when the proton is removed from either $\mathrm{CH}_{2}{ }^{+}$or $\mathrm{CH}^{+}$. The temporal 215 evolution of the kinetic energy released by the single fragments is shown in Figure S3 of the SI, 216 which indicates, that apart from the $\mathrm{C}-\mathrm{C}$ bond also the $\mathrm{C}-\mathrm{H}$ bond dissociates. The low energy 217 peak in Figure 4 follows precisely the evolution that was observed for the peak assigned to 218 dissociation in the two-body breakup in Figure 3. This constitutes strong evidence that we 219 observe the same cation dynamics in the two-body and in the three-body-breakup. The KER 220 spectra for the three-body breakup are too broad to clearly observe the high energy shoulder 221 associated with the launching of proton migration. However, since dissociation in the cationic 
222

223

224

225

226

227

228

229

230

231

232

233

234

235

236

237

238

239

240

241

242

243

244

245

246

states requires the absorption of a fifth photon, compared to the four photons required to initiate proton migration dynamics on the A-state, it is most likely that the latter process as well is initiated on the cation and probed via ionization to the triply charged states.

In the three-body breakup channel we have access to the direction in which the proton is emitted, allowing us to follow the proton migration process in detail. At its simplest, if the proton trajectory is close to the direction of the $\mathrm{CH}^{+}$fragment, it means that the molecule was close to the vinylidene isomer when Coulomb exploding. While, if the proton is close to the $\mathrm{C}^{+}$fragment direction, the molecule is closer to acetylene. Such an analysis allows for the construction of a molecular movie of the proton migration reaction, where key frames are represented by the Newton plots in Figure 5 as described in the figure caption. Since proton migration in the acetylene cation initiated on the A-state results in a bound vinylidene cation (i.e. non-dissociative), its Coulomb explosion will yield in a high KER. Therefore, the dynamics of interest were isolated by filtering the total three-body KER data, selecting only those correlated events above $13 \mathrm{eV}$, thereby excluding the $\mathrm{C}-\mathrm{C}$ bond dissociation events visible in Figure 3 and Figure 4. The cut-off for energy filtering is indicated as a black line in Figure 4a). For comparison, unfiltered Newton plots are presented in Figure S5 in the SI. In Figure 5 proton fragment momenta are presented in the molecular frame ${ }^{32}$, in concert with classically calculated values (shown as symbols) which assume Coulomb potentials and are included to serve as a visual guide. Despite substantial deviations of triply charged states from a Coulomb potential, the agreement between this simple calculation and the experimental data is very good. Starting from the linear geometry of neutral acetylene in the ground-state, the trans-configuration is reached after $20 \mathrm{fs}$, indicated by a depletion in signal at $(x=-1.0 \mid y=0.0)$ which is shown in blue and an increased signal at $(x=-0.3 \mid y=0.7)$ shown in green. This matches a half-period of the trans-bent vibrational mode. At 40 fs the centre of mass reaches the classically calculated transition state $(x=0.0 \mid y=1.0)$. This agrees with timescales predicted by our semi-classical 
247

248

249

250

251

252

253

254

255

256

257

258

259

260

261

262

263

264

265

266

267

268

269

270

simulations for a population transfer to the ground state mediated by the conical intersection. A maximum in vinylidene formation (classically calculated value $(x=0.6 \mid y=0.8))$ is observed around $100 \mathrm{fs}$, after which the population maximum swings back to the acetylene side (with a vinylidene population minimum around $150 \mathrm{fs}$ ). This oscillation continues, with the next vinylidene

population maximum observed at $180 \mathrm{fs}$. All time values are associated with an error of $\pm 5 \mathrm{fs}$.

The to and fro isomerization behaviour of Figure 5 is perfectly reproduced by the $a b$ initio trajectory simulations of the vibronic dynamics, as evinced in Figure 6. While a single recurrence had also been observed in $\mathrm{C}_{2} \mathrm{D}_{2}{ }^{2+12,13}$ we now see further oscillations associated with the evolution of the nuclear wavepacket. We deduce from these results the following proton migration process: Both, X-and A-states are populated in a three-, and four-photon process, respectively (Figure 1). According to our calculations and Ref. [22], the A-state population is depleted by roughly half in the first $40-50$ fs via a trans-bent conical intersection to hot vibrational states of the ground electronic state of $[\mathrm{HC}=\mathrm{CH}]^{+}$. Formation of vinylidene takes place exclusively here, where the isomerization barrier can be overcome in both directions, which is why we observe the to and fro isomerization between acetylene and vinylidene (see green arrow in Figure 1). The initial X-state population does not contribute to isomerization since its energy is too low to overcome the isomerization barrier. The isomerization process itself is 'primed` by the pre-eminence of motion along the trans-bending mode in the excited state (see Figures S6-S8 in the SI). The solid lines in Figure 6 represent the total ground-and excited-state populations of acetylene (red) and vinylidene (blue) cation, as elucidated in the SI. As evinced in Figure 6, the agreement is excellent up to $150 \mathrm{fs}$. From a single exponential fit we estimate the initial isomerization time to be approximately 41 fs (theory) and $43 \pm 10$ fs (experiment), in accord with previous results ${ }^{14}$. The temporal resolution achieved, results from the fact that both, the pump and the probe step are highly nonlinear (see Figure S2 in the SI). 
INITIAL STATE ASSIGNMENT - II. In order to validate the proposed interpretation of the

272 three-fragment correlation data, we confirm that the imaged dynamics occur on the state of

273 interest - i.e. the A-state of the cation. Evidence for a low-lying electronic state of $\Sigma$ symmetry

274 (such as the A-state) has already been presented in the single pulse experiments of Figure 1

275 with the appearance of a high energy shoulder analogous to observations at the FEL and its

276 angular dependence, which reveals ionization from the HOMO-1. While Coulomb explosion

277 occurs in the triply charged molecule $\mathrm{C}_{2} \mathrm{H}_{2}{ }^{3+}$, there exist multiple combinations of pump and

278 probe pulses that may be employed to reach this ionic state. To verify that it is indeed dynamics

279 on the cationic state that are being probed rather than the neutral or the subsequently prepared

280 dicationic surface, we first note that we expect negligible dicationic populations to be prepared

281 by the pump step since the ratio of parent ion $\mathrm{C}_{2} \mathrm{H}_{2}{ }^{+}$to $\mathrm{C}_{2} \mathrm{H}_{2}{ }^{2+}$ in the TOF spectrum is $>100: 1$,

282 demonstrating that the pump step strongly favours preparation of the cation over the dication.

283 Additionally, neither pump- nor probe-pulses alone contribute more than $0.3 \%$ to the correlated

284 counts of the two-body breakup, compared to their joined interaction. Of course, our method is

285 only sensitive to the combination of pump and probe step, and thus an extremely efficient (but

286 here undesirable) probe step for the transition from the $2^{+} \rightarrow 3^{+}$states, compared to the $1^{+} \rightarrow 2^{+}$

287 could in principle compensate for the pump efficiency. We therefore estimated the tunnel

288 ionization rates for these processes based on the Keldysh-theory (see section III. in the SI). The

289 results show the $1^{+} \rightarrow 2^{+}$ionization process to be $>100$ times more efficient than the $2^{+} \rightarrow 3^{+}$

290 ionization. Thus the limiting factor in the probe step is the ionization from $2^{+} \rightarrow 3^{+}-\mathrm{a}$ barrier

291 which is overcome, since we are observing the breakup of a triply charged molecule. These

292 calculations also suggest that $1^{+} \rightarrow 2^{+}$process is near saturation employing the laser intensities

293 used here. The latter point is consistent with (and perhaps a necessary precondition for) the

294 observation of the triply charged species. Combining the efficiencies of pump and probe step it 
appears therefore extremely unlikely that we observe dication dynamics in the case of three-

296 fragment correlation - which is also supported by the fact that the same dissociation dynamics

297 is observed both in the dication (Figure 3) and the trication (Figure 4), as discussed earlier.

298 Further, dynamics in neutral $\mathrm{C}_{2} \mathrm{H}_{2}$ can also be excluded on the basis of the power dependence 299 measurement of the single $266 \mathrm{~nm}$ pulse experiment presented in Figure S1 of the SI. While for 300 low pump powers we observe a four-photon absorption which matches the ionization potential 301 of the A-state energetically, for higher pump powers it is indicated that we are driving the pump 302 step in saturation. This saturation effect also holds for the experimental condition of the pump303 probe experiment. Thus, by the arrival time of the probe-pulse, there are no neutral molecules 304 left in the jet. Additionally, the identity of the KER of a $\mathrm{CH}^{+}$fragment from the two-body breakup 305 with an uncorrelated $\mathrm{CH}^{+}$fragment, as discussed in Figure $\mathrm{S} 4$ of the $\mathrm{SI}$, further indicates, that 306 we are probing dynamics in the cation. Finally, this assignment is strongly supported by the

307 excellent agreement with new high-level theoretical simulations of the $\mathrm{C}_{2} \mathrm{H}_{2}{ }^{+} \mathrm{A}$-state initiated 308 dynamics, as shown in Figure 6.

In summary, if one aims to populate the excited states of charged molecules whose difference in ionization potentials is too large to be overcome by $800 \mathrm{~nm}$ photons, our experimentally rather simple approach provides an alternative to demanding VUV sources.

312 Indeed, given the limited availability, repetition rate, pulse stability and timing jitter of FELs, our 313 approach provides tremendous benefits in terms of statistics and temporal resolution.

314 Combining pump-probe CEI with multiphoton absorption of UV light opens the door to time315 resolved imaging of chemical reactions and very rich dynamics since a variety of electronic 316 states is accessed simultaneously. Careful consideration, as here, makes it possible to identify 317 their origins unambiguously. Combined with theoretical simulations, our results present the most 318 complete picture of proton migration and $\mathrm{C}=\mathrm{C}$ bond breaking on the ground and excited states 319 of $\mathrm{C}_{2} \mathrm{H}_{2}{ }^{+}$to date. 
321 Experimental technique: Experiments were carried out at the Advanced Laser Light Source 322 ALLS (INRS-EMT, Varennes, Canada). We employ pump-probe CEl: A pump-pulse (266 nm, $32 \mathrm{fs}, 3.2 \mu \mathrm{J}$ ) ionizes the system to the cation and launches proton migration dynamics. A time delayed probe-pulse $(800 \mathrm{~nm}, 40 \mathrm{fs}, 44 \mu \mathrm{J})$ further ionizes it to higher charged states. Its electric field stripes-off electrons almost immediately, leaving positively charged fragments behind which undergo Coulomb explosion. They represent the molecule's geometric configuration at the

327 arrival time of the probe-pulse. The strength of CEI is its ability to directly image geometrical 328 structures. It does not provide direct observation of electronic states, as photoelectron spectroscopy would do. Nevertheless, we are able to draw conclusions about the electronic states populated in the initial ionization from the angular distribution of the molecular fragments 331 and theoretical support.

Intense $266 \mathrm{~nm}$ pulses were obtained by sum frequency generation of the fundamental beam ( $800 \mathrm{~nm}, 2.5 \mathrm{KHz}, 35 \mathrm{fs}$, KM labs) with its second harmonic (400 nm) in a $40 \mu \mathrm{m}$ thick type I BBO crystal (Altos Photonics). Pulse duration was controlled by chirped mirrors (Ultrafast Innovation) and/or fused silica plates from almost Fourier-transform limited (32 fs) to above 250 fs. Measurements were obtained with a homebuilt TG-FROG ${ }^{33}$. Pulse energy was varied 337 between $11.2 \mu \mathrm{J}$ and $3.2 \mu \mathrm{J}$. For the pump-probe experiments a 32 fs, $3.2 \mu \mathrm{J}$ pump-pulse of $338266 \mathrm{~nm}$ was combined with a $40 \mathrm{fs}, 44 \mu \mathrm{J}$ probe-pulse of $800 \mathrm{~nm}$. Laser polarization was 339 vertical for both, pump- and probe-beams. Laser pulses were focussed $(f=100 \mathrm{~mm})$ into a 340 collimated acetylene gas jet (Praxair AC 2.6AA-A5). Pump intensity on target was estimated to $3413 \times 10^{14} \mathrm{~W} / \mathrm{cm}^{2}$. Ionic fragments resulting from Coulomb explosion are collected with a uniform342 electric-field ion imaging spectrometer. Their full 3D momenta are retrieved using a time-and 343 position-sensitive delay line detector at the end of the $23 \mathrm{~cm}$ ion time of flight (TOF) 344 spectrometer (RoentDek Handels $\mathrm{GmbH}$ ). Due to the vertical polarisation direction two 
fragments of same mass and charge but opposite momenta (e.g. $\mathrm{CH}^{+} / \mathrm{CH}^{+}$, the acetylene channel) will hit the same detector position - but at different times. Thus choosing the correct TOF range enables us to distinguish them.

Due to a significantly increased intensity when pump- and probe-pulses overlap temporally, the effective focal volume is increased within which the threshold for ionization is reached, leading to an increase in ionized molecules. This allows determining time zero by the appearance of a $60 \%$ increase in total ion yield (sum of all ion fragments) when scanning the delay stage. Error of time zero assignment is around $\pm 5 \mathrm{fs}$, partially due to drifts over typical acquisition times of 24-48 h. Even though the pulse durations of $32 \mathrm{fs}$ and $40 \mathrm{fs}$ for pump-and probe-pulses appear quite long, we like to point out that both - pump and probe step - are highly non-linear. A fourphoton excitation effectively shortens the pump-pulse to $16 \mathrm{fs}$ and also the effective tunnel ionization time for the probe-pulse will be much shorter than the actual pulse duration.

Data analysis: Due to the large count rates produced in this experiment (typically $>3$ ions per laser shot), a sophisticated algorithm is used to parse the data for true coincidences ${ }^{34}$. A TOF window is defined in order to identify events registered by the position sensitive detector as specific ions - either $\mathrm{H}^{+}, \mathrm{C}^{+}, \mathrm{CH}^{+}, \mathrm{CH}_{2}{ }^{+}$, or $\mathrm{CC}^{+}$. The data is then parsed, laser shot by laser shot, for the existence of a specific fragmentation channel-e.g. $\left(\mathrm{H}^{+}, \mathrm{C}^{+}, \mathrm{CH}^{+}\right)$for the three-body breakup, or for the two-body breakup $\left(\mathrm{CH}^{+}, \mathrm{CH}^{+}\right)$as the acetylene channel and $\left(\mathrm{C}^{+}, \mathrm{CH}_{2}^{+}\right)$giving the vinylidene channel. If data from a laser shot yields the desired ions for the fragmentation channel, the momentum for these ions is calculated. If the momentum sum is near zero $\left(<10^{-23}\right.$ $\mathrm{kg} \mathrm{m} / \mathrm{s}$ ), the group of ions is considered a true coincidence, originating from the same molecule. The momentum information for this channel is stored for further calculation of various metrics e.g. KER, $\theta$ (see inset Figure 1), etc. It is possible with these high count rates that multiple ions are detected in the TOF windows defined for a specific channel, leading to several possible 
combinations of ions - the majority of which are false. In this case, all ion combinations are considered and only that which yields near zero momentum is stored as a true coincidence.

371 Theoretical methods: The excited nonadiabatic molecular dynamics on the $A^{2} \Sigma_{g}^{+}$state were 372 simulated using the full multiple spawning approach ${ }^{35}$ - a semiclassical method. In this 373 methodology, the vibronic wavepacket is represented in a basis of direct products (over each 374 Cartesian coordinate) of frozen Gaussian functions. These basis functions, or trajectories, are 375 propagated classically, with the potential energy surfaces determined "on-the-fly" using ab initio 376 electronic structure methods. The number of trajectories expands dynamically, to account for 377 regions of nonadiabatic coupling between electronic states, by "spawning" (approximating non378 adiabatic transitions) new basis functions onto different electronic states as needed. The 379 requisite energy gradients and derivative couplings required to propagate the trajectories were 380 computed at a very high level of electronic structure theory, employing atomic natural orbital 381 (ANO) basis sets and a first-order multireference configuration interaction (FO-MRCI) treatment 382 of electron correlation ${ }^{36}$. The underlying complete active space (CAS) reference functions for 383 the $\mathrm{MRCl}$ procedure were determined employing a 7 electron, 7 orbital (7e,7o) active space. 384 The initial distribution of nuclear positions and momenta for the simulation were generated by 385 386 sampling the ground vibrational state of neutral acetylene ${ }^{37}$. From 20 initial conditions, 1480 spawned trajectory basis functions were generated. On the basis of these simulations, the 387 adiabatic state populations as a function of time, as well as the geometric character of the 388 wavepacket (i.e. acetylenic vs. vinylidenic) could be readily determined. Details on the theoretical methods are provided in the supplementary information.

Assuming a Coulomb potential for the $3^{+}$charge state $\left(\mathrm{C}_{2} \mathrm{H}_{2}{ }^{3+} \rightarrow \mathrm{C}^{+}+\mathrm{CH}^{+}+\mathrm{H}^{+}\right)$, 391 momentum positions within the Newton plot have been calculated for four structures using 392 classical mechanics; (1) $\mathrm{C}_{2} \mathrm{H}_{2}$ at equilibrium, (2) $\mathrm{C}_{2} \mathrm{H}_{2}{ }^{+}$in the trans geometry, (3) $\mathrm{C}_{2} \mathrm{H}_{2}{ }^{+}$at the 393 transition state at the conical intersection, and (4) $\mathrm{C}_{2} \mathrm{H}_{2}{ }^{+}$in vinylidene geometry. For the $\mathrm{CH}^{+}$ 
394 fragment, it is assumed that the charge is located on the carbon with a mass of 13 . The initial

395 velocities of the fragments are assumed to be zero. Geometrical structures were obtained using

396 the level of theory described above.

397

\section{Acknowledgement}

399 The authors gratefully acknowledge supportive discussions with Dr. Michael Spanner and

400 research funding from NSERC, FQRNT and CIPI. H.I. acknowledges financial support from the

401 NSERC-Banting Postdoctoral Fellowships Program. S.B. and V.W. are thankful for financial

402 support from NSERC.

403 Author contribution:

404 H.I. and F.L. designed the experiment, H.I., B.W., S.B., B.E.S., N.T., E.B., M.G., C.H., and J-

405 C.K. carried out the experiments, M.S.S. and F.L. provided the theoretical support, H.I., B.W.,

406 S.B., V.W., M.S.S., J.S., and F.L. carried out data analysis and interpretation, H.I., F.L., M.S.

407 and J.S. wrote the manuscript, with support from all other authors.

408 Additional information

409 The authors declare no competing financial interests. Supplementary information

410 accompanies this paper. Correspondence and requests for materials should be addressed to

411 H. I. and F. L.

412 


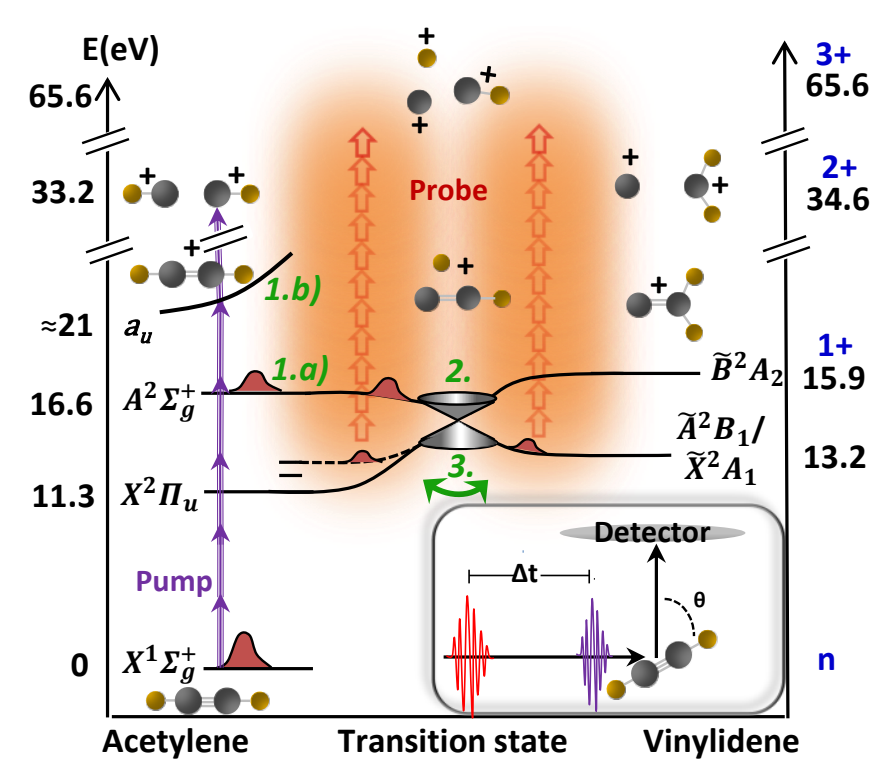

414 Figure 1 - Experimental scheme: (1.a) A $266 \mathrm{~nm}$ pump-pulse populates the first excited state $\boldsymbol{A}^{2} \boldsymbol{\Sigma}_{g}^{+}$of $[\mathrm{HC}=\mathrm{CH}]^{+}$in

415 a four-photon ionization process. Proton migration pathway: (2.) Within 40 fs the wavepacket enters a region of

416 strong nonadiabatic coupling with the ground $X^{2} \Pi_{u}$ state of $[\mathrm{HC}=\mathrm{CH}]^{+}(1+)$. Passage through this region involves

417 relaxation to the $\mathrm{X}$-state via a conical intersection. (3.) Following this transition, the molecule, now in a highly

418 vibrationally excited state, may undergo isomerization to the vinylidene cation $\left[\mathrm{C}=\mathrm{CH}_{2}\right]^{+}$. A to and fro isomerization

419 between acetylene and vinylidene takes places, indicated by the green double-arrow. Dissociation pathway: (1.b)

420 While the 32 fs pump-pulse is present, a fifth photon can be absorbed on the A-state thus exciting population to

421 higher lying states (presumably of $a_{u}$ symmetry, see Figure S9 in the SI) which have sufficient energy to dissociate

422 along the $\mathrm{C}=\mathrm{C}$ bond. Pictograms show the molecular structure in each configuration, starting from neutral acetylene

$423(n)$ in the lower left corner. After excitation, $[\mathrm{HC}=\mathrm{CH}]^{+}$remains in linear configuration $\left(\mathrm{D}_{\infty h}\right)$, reaches the trans

424 geometry (see Figure 5), followed by the transition state and changes to a "Y-shape" once a proton migrates from

425 one $\mathrm{C}$-atom to the other in the vinylidene structure $\left(\mathrm{C}_{2 v}\right)$. To probe the nuclear structure, either further $266 \mathrm{~nm}$

426 photons are absorbed to reach the dication of acetylene and vinylidene (single pulse experiment) or a second time

427 delayed $800 \mathrm{~nm}$ pulse leads to Coulomb explosion of the charged molecule (pump-probe experiment). With the latter

428 we reach the doubly charged (2+), as well as the triply charged ionic states (3+) and thus correlate either two

429 fragments $\left(\mathrm{CH}^{+}+\mathrm{CH}^{+}\right.$or $\left.\mathrm{C}^{+}+\mathrm{CH}_{2}^{+}\right)$or three fragments $\mathrm{H}^{+}+\mathrm{C}^{+}+\mathrm{CH}^{+}$with each other. In the inset a sketch of the

430 experimental setup with orientations of molecules, laser pulses and time of flight (TOF) direction towards the detector

431 is given, with $\theta$ being the angle between molecular axis and laser polarization. Molecules being oriented parallel to

432 the laser field correspond to $\theta=0^{\circ}$. Energy levels are taken from Ref. ${ }^{27}$ and our calculations. 

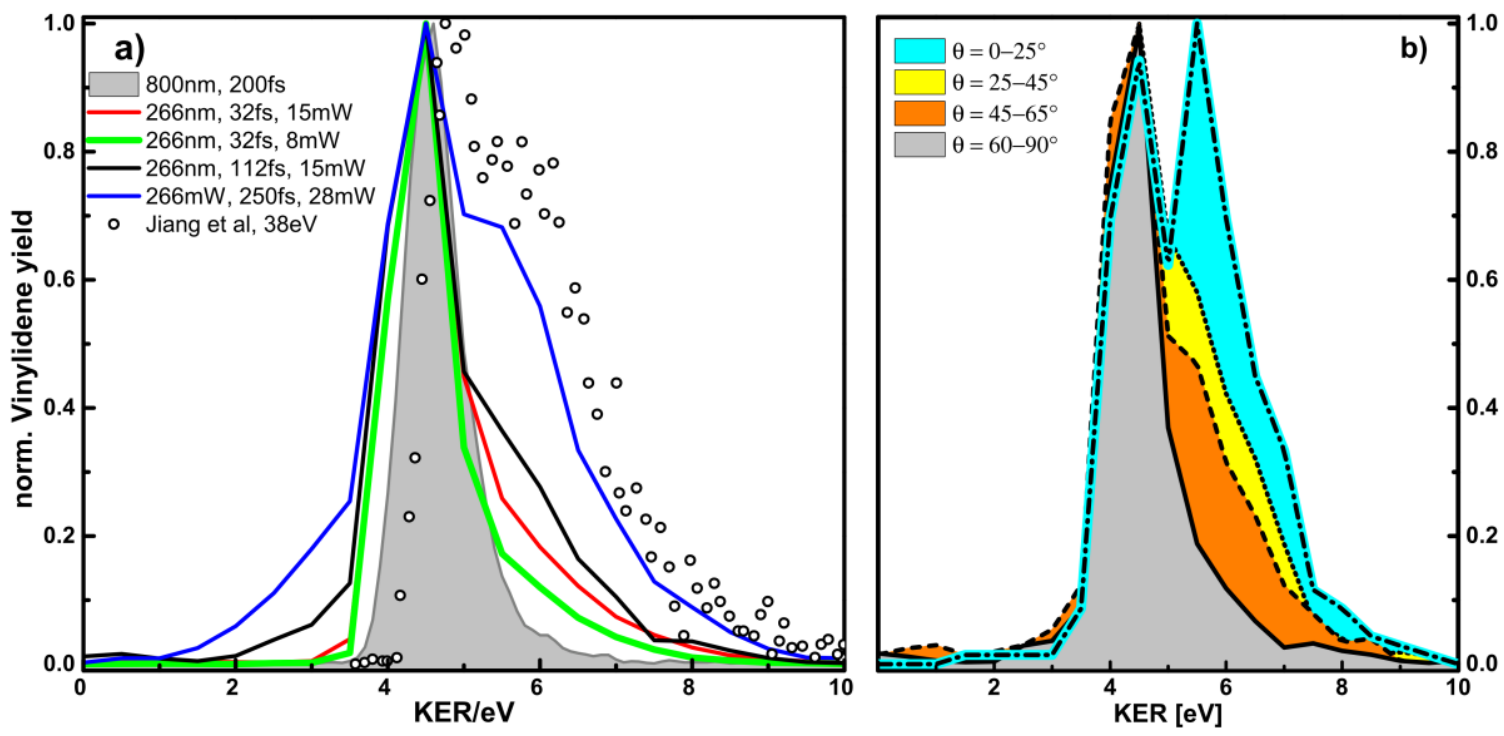

433

434 Figure 2 - single pulse experiment: a) Wavelength-, pulse duration- and pulse power dependence on the

435 vinylidene yield. Filled grey curve: $800 \mathrm{~nm}$ excitation, $200 \mathrm{fs}$ pulse duration, $1 \cdot 10^{8} \mathrm{~W}$, green curve: $266 \mathrm{~nm}$ excitation,

43632 fs pulse duration, $1 \cdot 10^{8} \mathrm{~W}$, red curve: $266 \mathrm{~nm}$ excitation, 32 fs pulse duration, $2 \cdot 10^{8} \mathrm{~W}$, black curve: $266 \mathrm{~nm}$

437 excitation, 110 fs pulse duration, $0.5 \cdot 10^{8} \mathrm{~W}$, blue curve: $266 \mathrm{~nm}$ excitation, 250 fs pulse duration, $0.4 \cdot 10^{8} \mathrm{~W}$ open

438 circles: excitation with $38 \mathrm{eV}$ from an $\mathrm{FEL}^{14}$. b) Cuts of the angular distribution of the $266 \mathrm{~nm}, 110 \mathrm{fs}$ spectrum shown

439 as black solid line in a). Cyan corresponds to angles between $0-25^{\circ}$ (note that the high energy peak exceeds the

440 main peak here), yellow corresponds to $25-45^{\circ}$, orange to $45-60^{\circ}$ and grey to $60-90^{\circ}$. All curves are normalized to the

441 peak maximum.

442

443 


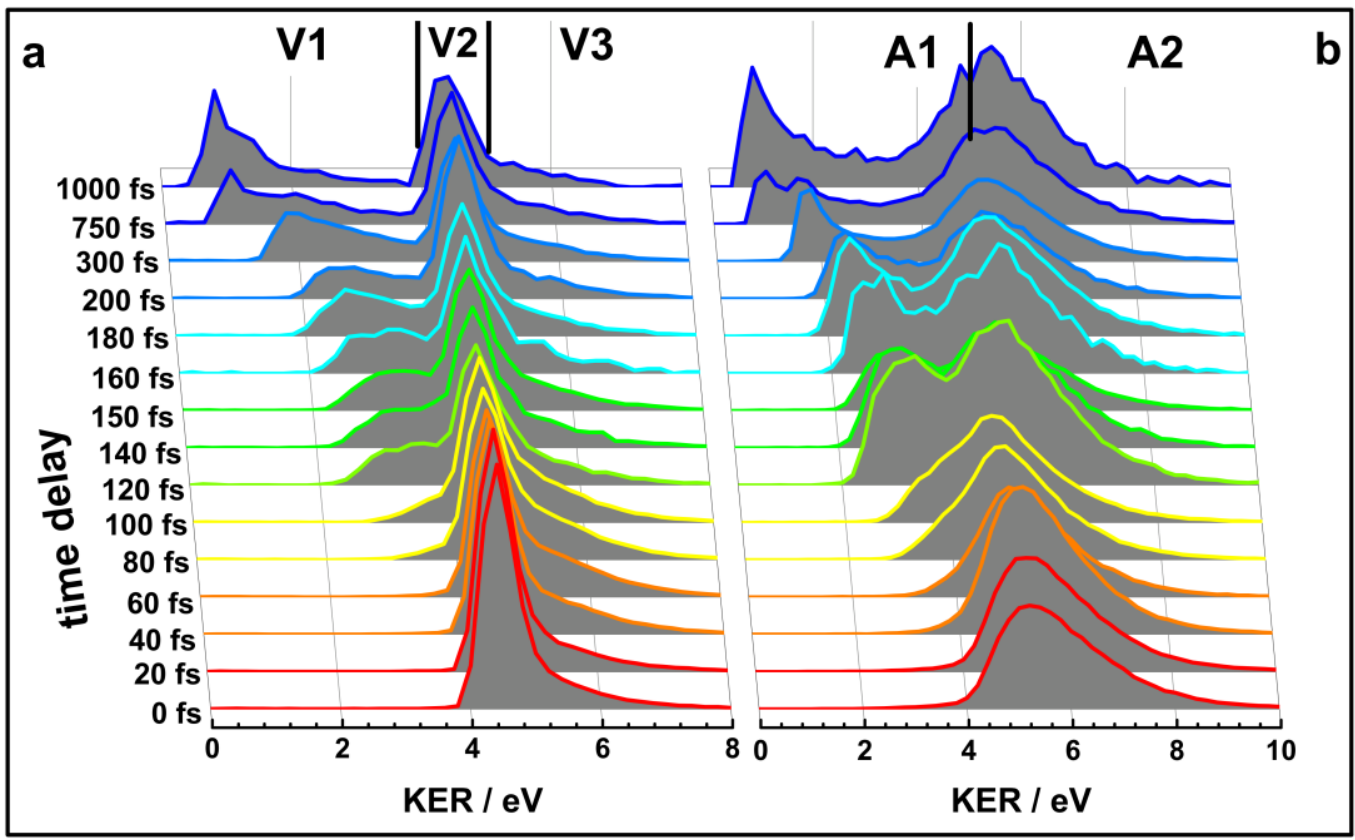

445 Figure 3 - Two-fragment correlation-Kinetic energy release: Vinylidene channel $\mathrm{C}^{+}+\mathrm{CH}_{2}{ }^{+}$is shown in a and

446 acetylene channel $\mathrm{CH}^{+}+\mathrm{CH}^{+}$in $\mathbf{b}$ with increasing time delay from 0 fs (red) to 1000 fs (blue). The vinylidene spectrum

447 contains three different regions: $\mathrm{V} 1$ : the low energy range below $4 \mathrm{eV}, \mathrm{V} 2$ : the intermediate energy range between 4

448 and $5 \mathrm{eV}$ and $\mathrm{V} 3$ : the high-energy range, $>5 \mathrm{eV}$. The acetylene channel is divided into two regions, the low energy

449 region $\mathrm{A} 1:<4 \mathrm{eV}$ and the high-energy region $\mathrm{A} 2:>4 \mathrm{eV}$. Spectra are normalized by the number of correlated counts,

450 to account for varying acquisition times at different time-steps, as well as for minor fluctuations in pulse power or gas

451 pressure. This normalization allows comparison of the absolute count number in each channel at each time delay. 


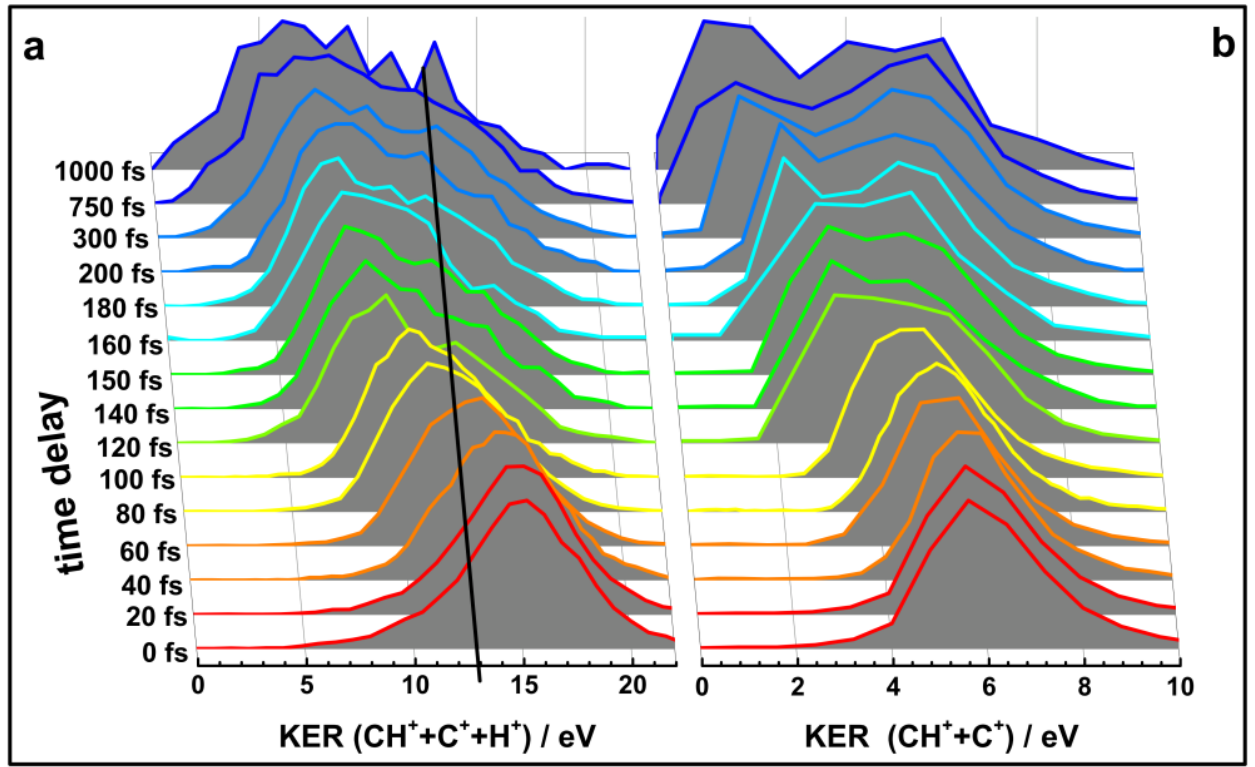

455 Figure 4 - Three-fragment-correlation data-Kinetic energy release: Peak values are normalized to the maximum.

456 a) total $\mathrm{KER} \mathrm{CH}^{+}+\mathrm{C}^{+}+\mathrm{H}^{+}$, b) $\mathrm{KER}$ from $\mathrm{CH}^{+}+\mathrm{C}^{+}$fragments. The black line in a) indicates the energy cut-off for the

457 Newton plots of Figure 5. Binning was adjusted according to the number of correlated counts available for each time 458 delay.

459

460

461 


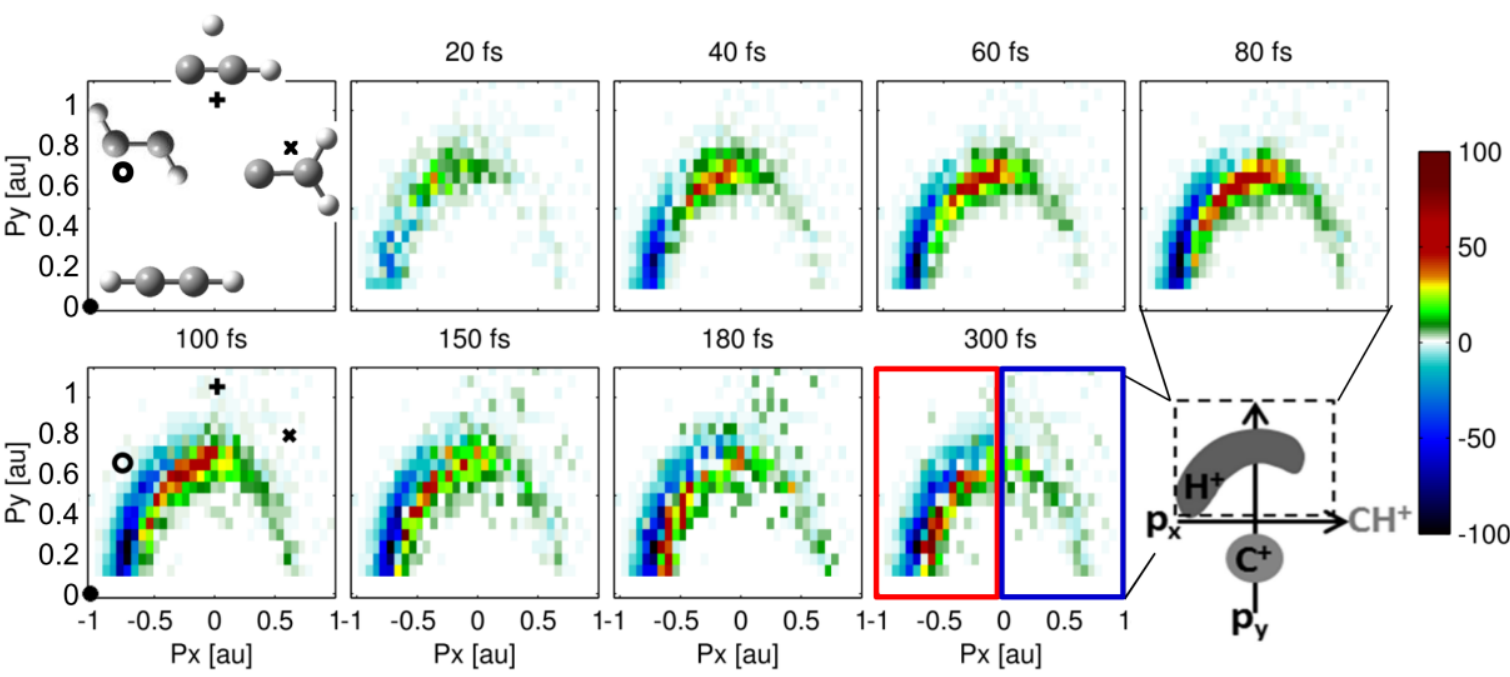

Figure 5 - The molecular movie of proton migration: Newton plots filtered for KER $>13 \mathrm{eV}$. Symbols show

464 classically calculated results assuming Coulomb potentials for linear configuration (·), trans configuration (o), transition state $(+)$ and vinylidene $(x)$, given in the upper left corner. As indicated on the lower right, all molecules are rotated such that the momentum vector of $\mathrm{CH}^{+}$points towards the positive $\mathrm{x}$-axis, the relative momentum of $\mathrm{C}^{+}$is

467 confined to the negative $y$-axis. Here, we plot only data of the momentum vector of $\mathrm{H}^{+}$, after subtracting the

468 distribution at $\Delta \mathrm{t}=0$. The plots show the evolution of $\mathrm{H}^{+}$momenta with increasing time delay from 0 fs to 300 fs,

469 normalized to the integral. The distribution appears localized at $20 \mathrm{fs}$ (dark green data points) and spreads out 470 towards the $\mathrm{CH}^{+}$ion with increasing time delay. $\mathrm{C}^{+}$is considerably heavier than $\mathrm{H}^{+}$and thus localized, while the light

$471 \mathrm{H}^{+}$propagates with time being transferred from the acetylene configuration to the vinylidene one. White colours

472 correspond to zero, blue colours to negative contributions (after subtraction, i.e. where the population originates) and 473 other colours to positive contributions (i.e. where the population is going). 
475

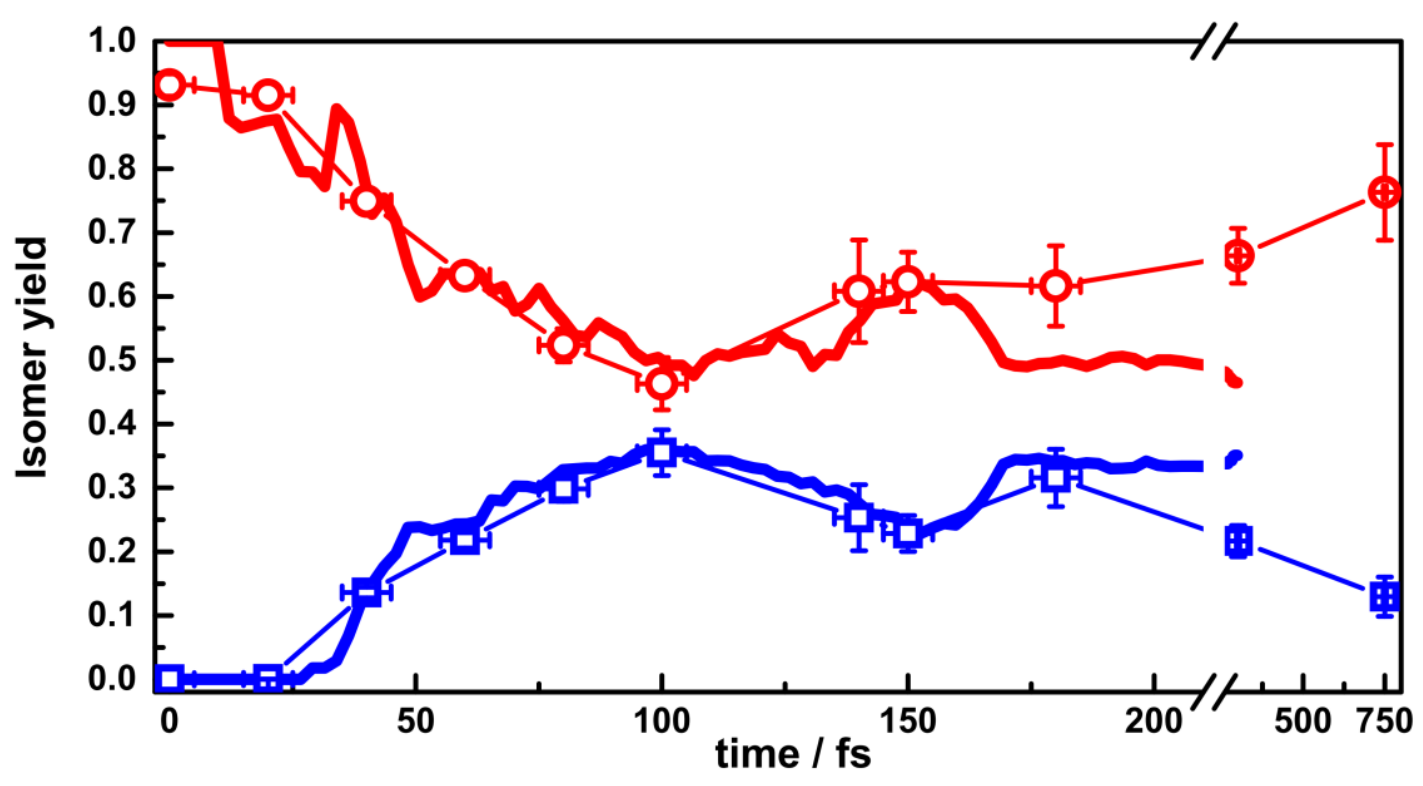

476 Figure 6 - Comparison of theory (thick lines) and experiment (open symbols) for acetylene (red) and

477 vinylidene (blue) yield: Theoretical curves show the superposition of acetylene and vinylidene yield in both the

478 ground state and excited states of the cation as a function of time (see Figure S8 in the SI for details). Experimental

479 values are obtained by integrating the Newton plots of Figure 5 for negative $\mathrm{P}_{\mathrm{x}}$ (acetylene, circles) or positive $\mathrm{P}_{\mathrm{x}}$

480 (vinylidene, squares), as indicated there by the red and blue box at $300 \mathrm{fs}$. Note the broken axis for long time delays.

481 Experimental data are corrected by offset and scaling factor to fit the theoretical points. 
485

486

487

488

489

490

491

492

493

494

495

496

497

498

499

500

501

502

503

504

505

506

507

508

509

510

511

512

513

514

515

1. Zewail, A. H. Four-dimensional electron microscopy. Science 328, 187-93 (2010).

2. Weinstein, J. A. \& Hunt, N. T. In search of molecular movies. 4, 4-5 (2012).

3. Blaga, C. I. et al. Imaging ultrafast molecular dynamics with laser-induced electron diffraction. Nature 483, 194-7 (2012).

4. Haessler, S. et al. Attosecond imaging of molecular electronic wavepackets. Nat. Phys. 6, 200-206 (2010).

5. Wörner, H. J., Bertrand, J. B., Kartashov, D. V, Corkum, P. B. \& Villeneuve, D. M. Following a chemical reaction using high-harmonic interferometry. Nature 466, 604-7 (2010).

6. Chapman, H. N. et al. Femtosecond X-ray protein nanocrystallography. Nature 470, 7377 (2011).

7. Miller, R. J. D. et al. "Making the molecular movie": first frames. Acta Crystallogr. A. 66, 137-56 (2010).

8. Eichberger, M. et al. Snapshots of cooperative atomic motions in the optical suppression of charge density waves. Nature 468, 799-802 (2010).

9. Barty, A., Küpper, J. \& Chapman, H. N. Molecular imaging using X-ray free-electron lasers. Annu. Rev. Phys. Chem. 64, 415-35 (2013).

10. Légaré, F. et al. Imaging the time-dependent structure of a molecule as it undergoes dynamics. Phys. Rev. A 72, 1-4 (2005).

11. Bocharova, I. et al. Charge Resonance Enhanced Ionization of CO_\{2\} Probed by Laser Coulomb Explosion Imaging. Phys. Rev. Lett. 107, 63201 (2011).

12. Matsuda, A., Fushitani, M., Takahashi, E. J. \& Hishikawa, A. Visualizing hydrogen atoms migrating in acetylene dication by time-resolved three-body and four-body Coulomb explosion imaging. Phys. Chem. Chem. Phys. 13, 8697-704 (2011).

13. Hishikawa, A., Matsuda, A., Fushitani, M. \& Takahashi, E. J. Visualizing Recurrently Migrating Hydrogen in Acetylene Dication by Intense Ultrashort Laser Pulses. Phys. Rev. Lett. 99, 258302 (2007).

14. Jiang, Y. H. et al. Ultrafast Extreme Ultraviolet Induced Isomerization of Acetylene Cations. Phys. Rev. Lett. 105, 263002 (2010).

15. Koopmans, T. Über die Zuordnung von Wellenfunktionen und Eigenwerten zu den Einzelnen Elektronen Eines Atoms. Physica 1, 104-113 (1934). 
16. McFarland, B. K., Farrell, J. P., Bucksbaum, P. H. \& Gühr, M. High harmonic generation from multiple orbitals in N2. Science 322, 1232-5 (2008).

17. Akagi, $\mathrm{H}$. et al. Laser tunnel ionization from multiple orbitals in $\mathrm{HCl}$. Science 325, 1364-7 (2009).

18. Smirnova, O. et al. High harmonic interferometry of multi-electron dynamics in molecules. Nature 460, 972-7 (2009).

19. Tilborg, J. Van et al. Femtosecond isomerization dynamics in the ethylene cation measured in an EUV-pump NIR-probe configuration. J. Phys. B At. Mol. Opt. Phys. 42, 081002 (2009).

20. Takahashi, E. J., Lan, P., Mücke, O. D., Nabekawa, Y. \& Midorikawa, K. Attosecond nonlinear optics using gigawatt-scale isolated attosecond pulses. Nat. Commun. 4, 2691 (2013).

21. Tzallas, P., Skantzakis, E., Nikolopoulos, L. a. a., Tsakiris, G. D. \& Charalambidis, D. Extreme-ultraviolet pump-probe studies of one-femtosecond-scale electron dynamics. Nat. Phys. 7, 781-784 (2011).

22. Madjet, M. E., Li, Z. \& Vendrell, O. Ultrafast hydrogen migration in acetylene cation driven by non-adiabatic effects. J. Chem. Phys. 138, 094311 (2013).

23. Douguet, N., Rescigno, T. N. \& Orel, a. E. Time-resolved molecular-frame photoelectron angular distributions: Snapshots of acetylene-vinylidene cationic isomerization. Phys. Rev. A 86, 013425 (2012).

24. Jiang, Y. H. et al. Ultrafast dynamics in acetylene clocked in a femtosecond XUV stopwatch. J. Phys. B At. Mol. Opt. Phys. 46, 164027 (2013).

25. Wells, E. et al. Adaptive strong-field control of chemical dynamics guided by threedimensional momentum imaging. Nat. Commun. 4, 2895 (2013).

26. Alnaser, A. et al. Momentum-imaging investigations of the dissociation of $D 2+$ and the isomerization of acetylene to vinylidene by intense short laser pulses. J. Phys. B At. Mol. Opt. Phys. 39, S485-S492 (2006).

27. Boyé-Péronne, S., Gauyacq, D. \& Liévin, J. Vinylidene-acetylene cation isomerization investigated by large scale ab initio calculations. J. Chem. Phys. 124, 214305 (2006).

28. Lezius, M. et al. Nonadiabatic Multielectron Dynamics in Strong Field Molecular Ionization. Phys. Rev. Lett. 86, 51-54 (2001).

29. Madjet, M. E.-A., Vendrell, O. \& Santra, R. Ultrafast Dynamics of Photoionized Acetylene. Phys. Rev. Lett. 107, 263002 (2011). 
30. Zyubina, T. S., Dyakov, Y. a, Lin, S. H., Bandrauk, a D. \& Mebel, a M. Theoretical study of isomerization and dissociation of acetylene dication in the ground and excited electronic states. J. Chem. Phys. 123, 134320 (2005).

31. Xu, H. et al. Two-proton migration in 1,3-butadiene in intense laser fields. Phys. Chem. Chem. Phys. 12, 12939-42 (2010).

32. Wales, B. et al. Multiple ionization and complete fragmentation of OCS by impact with highly charged ions Ar 4+ and Ar 8+ at $15 \mathrm{keV} \mathrm{q-1.} \mathrm{J.} \mathrm{Phys.} \mathrm{B} \mathrm{At.} \mathrm{Mol.} \mathrm{Opt.} \mathrm{Phys.} \mathrm{45,}$ 045205 (2012).

33. Schmidt, B. E. et al. Poor man's source for sub 7 fs: a simple route to ultrashort laser pulsesand their full characterization. Opt. Express 16, 18910-18921 (2008).

34. Wales, B. et al. A coincidence detection algorithm for improving detection rates in Coulomb Explosion Imaging. Nucl. Instruments Methods Phys. Res. Sect. A Accel. Spectrometers Detect. Assoc. Equip. 667, (2011).

35. Ben-Nun, M. \& Martínez, T. J. Ab Initio Quantum Molecular Dynamics. Adv. Chem. Phys. 121, 439-512 (2002).

36. Lischka, H. et al. COLUMBUS, an ab initio electronic structure program, release 7.0. (2012). at <http://www.univie.ac.at/columbus/>

37. J.F. Stanton, J. Gauss, M.E. Harding, P. G. S. et al. CFOUR, a quantum chemical program package written by: at <For the current version, see http://www.cfour.de.> 